\title{
Corrigendum
}

\section{Corrigendum to "Comparison of Some Tests of Fit for the Inverse Gaussian Distribution"}

\author{
D. J. Best, ${ }^{1}$ J. C. W. Rayner, ${ }^{1,2}$ and O. Thas ${ }^{2,3}$ \\ ${ }^{1}$ School of Mathematical and Physical Sciences, University of Newcastle, NSW 2308, Australia \\ ${ }^{2}$ National Institute for Applied Statistics Research Australia (NIASRA), School of Mathematics and Applied Statistics, \\ University of Wollongong, Wollongong, NSW 2522, Australia \\ ${ }^{3}$ Department of Mathematical Modelling, Statistics and Bioinformatics, Ghent University, Coupure Links 653, 9000 Gent, Belgium
}

Correspondence should be addressed to J. C. W. Rayner; john.rayner@newcastle.edu.au

Received 21 June 2016; Accepted 29 September 2016

Copyright (C) 2016 D. J. Best et al. This is an open access article distributed under the Creative Commons Attribution License, which permits unrestricted use, distribution, and reproduction in any medium, provided the original work is properly cited.

In the article titled "Comparison of some tests of fit for the Inverse Gaussian distribution" [1], there were a number of typographical and other errors. Except for the minor typographical error in $V_{0}$ below, all calculations reported appear to be correct.

In $(2.1)+$ sign in the denominator should be omitted so that the denominator is $24(\cdots)(\cdots)$. Also a hat is missing on $\phi$ and the last $\widehat{\phi}$ should not be cubed. Thus

$$
\begin{aligned}
\widehat{V}_{3}^{2} & =\frac{n \widehat{\phi}^{6}}{24(4+\widehat{\phi})\left(120+75 \widehat{\phi}+15 \widehat{\phi}^{2}+\widehat{\phi}^{3}\right)} \\
& \times\left\{\widehat{\widehat{Z}}^{3}(4+\widehat{\phi})-\overline{\widehat{Z}}^{2}\left(\frac{60}{\widehat{\phi}}+30+4 \widehat{\phi}\right)+\frac{120}{\widehat{\phi}^{3}}+\frac{195}{\widehat{\phi}^{2}}\right. \\
& \left.+\frac{123}{\widehat{\phi}}+32+3 \widehat{\phi}\right\}^{2} .
\end{aligned}
$$

In (2.2) the first occurrence of $1+$ should be $1-$. Thus

$V_{0}$

$$
\begin{aligned}
= & \frac{1}{n} \sum_{j, k=1}^{n} Z_{j k}^{-1} \\
& -2 \sum_{j=1}^{n} Z_{j}^{-1}\left\{1-\sqrt{\frac{\pi \widehat{\phi}}{2 Z_{j}}} \operatorname{erfce}\left(\frac{\left(Z_{j}+1\right) \sqrt{\hat{\phi}}}{\sqrt{2 Z_{j}}}\right)\right\} \\
& +\frac{n(1+2 \widehat{\phi})}{4 \widehat{\phi}} .
\end{aligned}
$$

In (2.5) and (2.6) $n$ s before $e_{3}$ and $e_{4}$ should both be deleted. Thus

$$
\begin{aligned}
& \pi_{3}(z)=\frac{\left(z^{3}-a_{3} z^{2}-b_{3} z-c_{3}\right)}{\sqrt{ } e_{3}}, \\
& \pi_{4}(z)=\frac{\left(z^{4}+a_{4} z^{3}+b_{4} z^{2}+c_{4} z+d_{4}\right)}{\sqrt{ } e_{4}} .
\end{aligned}
$$

In the definition of $e_{4}$ following (2.6) -253440 should be +253440 .

In example (ii) the value of $V_{0}$ should be 0.0033 .

\section{References}

[1] D. J. Best, J. C. W. Rayner, and O. Thas, "Comparison of some tests of fit for the inverse Gaussian distribution," Advances in Decision Sciences, vol. 2012, Article ID 150303, 9 pages, 2012. 


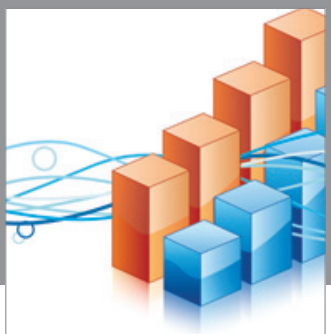

Advances in

Operations Research

vatem alat4

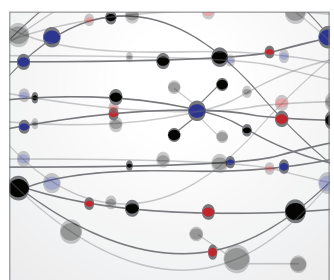

\section{The Scientific} World Journal
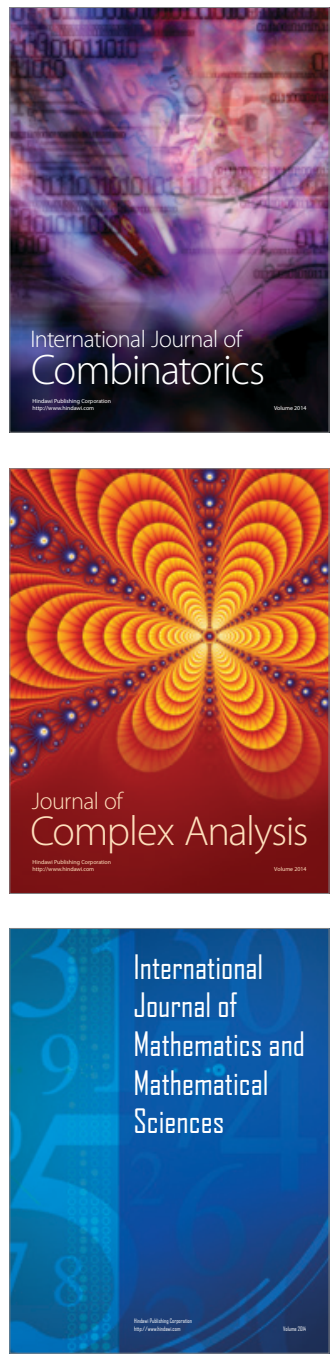
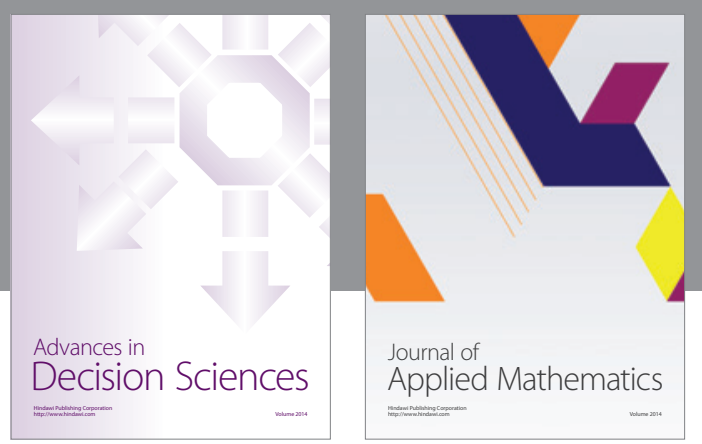

Algebra

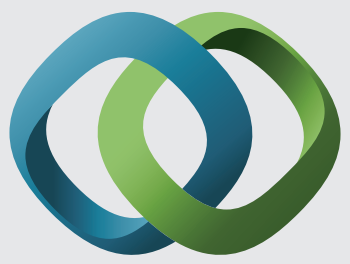

\section{Hindawi}

Submit your manuscripts at

http://www.hindawi.com




Mathematical Problems in Engineering


Journal of

Function Spaces

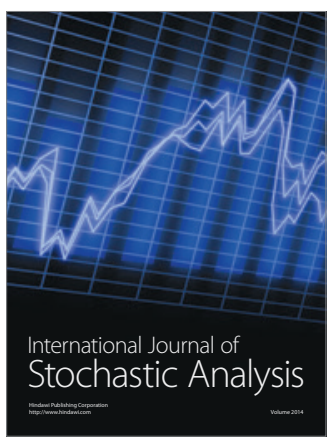

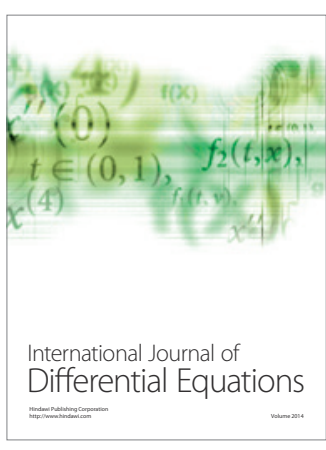
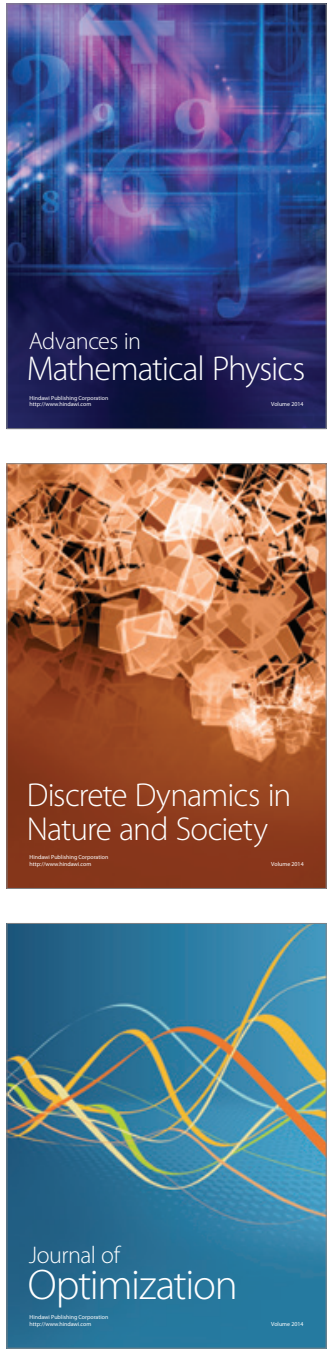